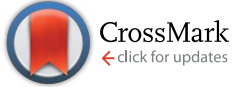

Cite this: RSC Adv., 2017, 7, 12908

Received 6th January 2017

Accepted 7th February 2017

DOI: $10.1039 / \mathrm{c} 7 \mathrm{ra00237h}$

rsc.li/rsc-advances

\section{Selective separation of oil and water with special wettability mesh membranes $\uparrow$}

\author{
Defei Liu, ${ }^{\text {abc }}$ Yuanlie $\mathrm{Yu}^{\mathrm{b}}$ Xin Chen ${ }^{\mathrm{c}}$ and Yuying Zheng ${ }^{\star a}$
}

Due to the different interfacial effects of oil and water, utilizing the special wettability of solid surfaces to design an oil and water separation process has been demonstrated to be an effective approach for oil/water separation. In this report, a simple process has been developed to fabricate special surface wettability mesh membranes. The carbon nanoparticles with diameters of $10 \mathrm{~nm}$ were first coated onto the surface of steel wires based on a candle soot coating process. These templates of carbon nanoparticles were then coated with a more stable layer of silica $\left(\mathrm{SiO}_{2}\right)$ particles via a facile chemical vapor deposition route. After being modified by two separate methods, a superhydrophobic/superoleophilic membrane was obtained by the use of $1 H, 1 H, 2 H, 2 H$-perfluorooctyltrichlorosilane (PFOTS) and a oleophobic/superhydrophilic membrane was obtained by using poly(diallyldimethylammonium-perfluorooctanoate) (PDDA-PFO). Separation experiments show that these superhydrophobic/superoleophilic or oleophobic/superhydrophilic mesh membranes can be used to selectively separate oil/water with a high flux of more than $930 \mathrm{~L} \mathrm{~m}^{-2} \mathrm{~h}^{-1}$ and a collecting efficiency of over $97 \%$. Furthermore, the repetitions of the separation experiments demonstrate that these superhydrophobic/superoleophilic or oleophobic/superhydrophilic mesh membranes are durable, stable and reusable, making them encouraging candidates for practical oil-polluted water treatment.

\section{Introduction}

With the development of modern industries, more and more oily wastewater is being generated and finding a viable processes to tackle this issue is required. ${ }^{1}$ Moreover, recent frequent oil spill events have further highlighted the necessity for effective oil and water separation. ${ }^{2}$ So far, it has been a worldwide challenge to effectively remove the oil or the water from such oily wastewater. Although traditional methods, such as oil skimmers, centrifuges, coalesces, settling tanks, depth filters, magnetic separations, flotation technologies, ignition of the oil and absorption can remove the oil from such oily wastewater, ${ }^{3,4}$ these methods usually occupy large space and are too complicated to operate because of time consumption or the use of additional chemicals or gases. ${ }^{3-5}$ Therefore, there is an increasing demand for the development of effective and inexpensive approaches for the clean-up of oil and water mixtures. ${ }^{6}$

The oil/water mixture separation is governed by different interfacial effects of oil and water. Hence, the use of special

${ }^{a}$ School of Chemical Engineering and Light Industry, Guangdong University of Technology, Guangzhou 510006, China.E-mail: zyyzhengyuying@163.com

${ }^{b}$ Advanced Membrane and Porous Materials Center, Chemical and Biological Engineering, King Abdullah University of Science and Technology, Thuwal 239556900, Saudi Arabia

${ }^{c}$ School of Environment and Chemical Engineering, Foshan University, Foshan 528000, China

$\uparrow$ Electronic supplementary information (ESI) available. See DOI: 10.1039/c7ra00237h wettability of solid surfaces to design an oil and water separation process has been considered as an effective approach. In order to exploit this special wettability to separate oil/water mixtures, potential separation materials should simultaneously possess an opposite wettability to water and oil. Therefore, the materials with special surface wettability, such as superhydrophobicity/ superoleophilicity or superoleophobicity/superhydrophilicity, have attracted considerable attention. To date, various superhydrophobic/superoleophilic or superoleophobic(oleophobic)/superhydrophilic materials, such as carbonaceous nanofiber hydrogels and aerogels, ${ }^{7-10}$ sponges, ${ }^{11-13}$ polymer, ${ }^{14-17}$ metals, ${ }^{18,19}$ metal oxides and hydroxides, ${ }^{20-23}$ boron nitride based materials, ${ }^{24,25}$ zeolite, ${ }^{26-28}$ graphene oxides ${ }^{29,30}$ and diamond-like carbon ${ }^{31}$ have been developed. Moreover, some of these materials have been adopted as membranes for oil/water separation. ${ }^{\mathbf{8}, \mathbf{1 4 - 1 8}}$

Principally, these membranes' surface wettability are dictated by surface roughness and its chemical composition. ${ }^{32,33}$ Consequently, artificial superhydrophobic/superoleophilic ${ }^{34}$ or superoleophobic(oleophobic)/superhydrophilic ${ }^{35}$ membranes for the separation of oil and water are usually prepared through a two-step process: creating a hierarchically rough surface on the membrane and then chemically modifying the membrane with low surface energy materials. Up to now, a number of methods have been put forward to increase the roughness of the membrane surface (e.g. laser, ${ }^{36}$ sol-gel process, ${ }^{37}$ chemical vapor deposition, ${ }^{38,39}$ and electrochemical method ${ }^{40}$ ). However, most of these methods lead to the formation of unstable layers, 
require expensive elements, rigorous reaction conditions, or involve complex processes.

In this work, we use carbon nanoparticles as templates following coat a layer of hydrophilic silica $\left(\mathrm{SiO}_{2}\right)$ particles on the surface of stainless steel mesh to form a stable hydrophilic/ oleophilic hierarchically rough $\mathrm{SiO}_{2} /$ carbon layer, fitting for making both superhydrophobic/superoleophilic and oleophobic/superhydrophilic membranes. Then, we modified the $\mathrm{SiO}_{2} /$ carbon stainless steel mesh in two ways. The first method is with $1 H, 1 H, 2 H, 2 H$-perfluorooctyltrichlorosilane (PFOTS) and the second is with poly(diallyldimethylammoniumperfluorooctanoate) (PDDA-PFO) to form superhydrophobic/ superoleophilic and oleophobic/superhydrophilic membranes, respectively. Static water and oil contact angles (CAs) were used to examine the superhydrophobicity/superoleophilicity or oleophobicity/superhydrophilicity of the as-prepared membrane samples. Because of the contrary surface wettability, the superhydrophobic/superoleophilic and oleophobic/superhydrophilic $\mathrm{SiO}_{2}$ /carbon stainless steel mesh membranes were used for the selective removal of oil or water from different oil/water mixtures. For oil enriched oil/water mixtures, superhydrophobic/superoleophilic mesh membranes can be selected to remove water from such oil/water mixtures. Otherwise, oleophobic/superhydrophilic mesh membranes can be selected to remove oil from water enriched oil/water mixtures. Finally, we studied the durability, stability and recyclability of these materials. Owing to simplicity of the preparation method, these superhydrophobic/superoleophilic or oleophobic/ superhydrophilic $\mathrm{SiO}_{2} /$ carbon stainless steel mesh membranes are low cost and can be easily scaled up, and will be promising and practical candidates for oil-polluted water treatment.

\section{Experimental}

\subsection{Materials}

Stainless steel mesh (350 mesh, 316L) and commercial candles were purchased from a supermarket. Tetraethyl orthosilicate (TEOS), ammonium hydroxide solution (28\%), PFOTS, PFO acid $\left(\mathrm{CF}_{3}\left(\mathrm{CF}_{2}\right)_{6} \mathrm{COOH}\right)$ and PDDA $\left(M_{\mathrm{w}}=100000-200000\right)$ were purchased from Sigma-Aldrich. Na-PFO (0.10 M) was prepared by reacting PFO acid with sodium hydroxide in water. All the chemicals were used as received without further purification.

\subsection{Preparation of the superhydrophobic/superoleophilic and oleophobic/superhydrophilic stainless steel mesh}

A stainless steel meshes (350 mesh, 316L) were place above the wick and soot was collected on the surface. And $10 \mathrm{~nm}$ carbon nanoparticles (Fig. S1 $\dagger$ ) were coated onto the stainless steel meshes (Fig. 1a and S.1.1†). Then we used a chemical vapor deposition method to form a more stable layer of $\mathrm{SiO}_{2} /$ carbon on the stainless steel meshes (Fig. $1 \mathrm{~b}$ and S.1.2†). Finally, the $\mathrm{SiO}_{2} /$ carbon stainless steel mesh were modified with PFOTS (Fig. 1d and S.1.3†) and PDDA-PFO (Fig. 1e and S.1.4†) respectively to form the superhydrophobic/superoleophilic and oleophobic/superhydrophilic mesh membrane.

\subsection{Separation flux, efficiency and stability tests of PFOTS or PDDA-PFO modified $\mathrm{SiO}_{2} /$ carbon stainless steel mesh membrane}

For PFOTS modified superhydrophobic/superoleophilic $\mathrm{SiO}_{2} /$ carbon stainless steel mesh membrane separation test, the oil/ water mixture was prepared by mixing $20 \mathrm{~mL}$ water (dyed blue with methyl blue) and $30 \mathrm{~mL}$ diesel oil. For PDDA-PFO modified oleophobic/superhydrophilic $\mathrm{SiO}_{2} /$ carbon stainless steel mesh membrane separation test, the oil/water mixture was prepared by mixing $30 \mathrm{~mL}$ water and $20 \mathrm{~mL}$ diesel oil (dyed red with Sudan III). During the separation process, one of the two modified membranes was placed between the two glass tubes corresponding to its modification (with a well-polished flat surface to provide an excellent seal). A similar setup was produced for the other membrane and its oil/water mixture. Both the meshes and tubes were clamped together. For every separation, the prepared oil/water mixtures were slowly poured into the upper glass tube and separated by the PFOTS or PDDA$\mathrm{PFO}$ modified $\mathrm{SiO}_{2} /$ carbon stainless steel mesh membranes. To test the stability of the PFOTS or PDDA-PFO modified $\mathrm{SiO}_{2} /$ carbon stainless steel mesh membranes, each separation

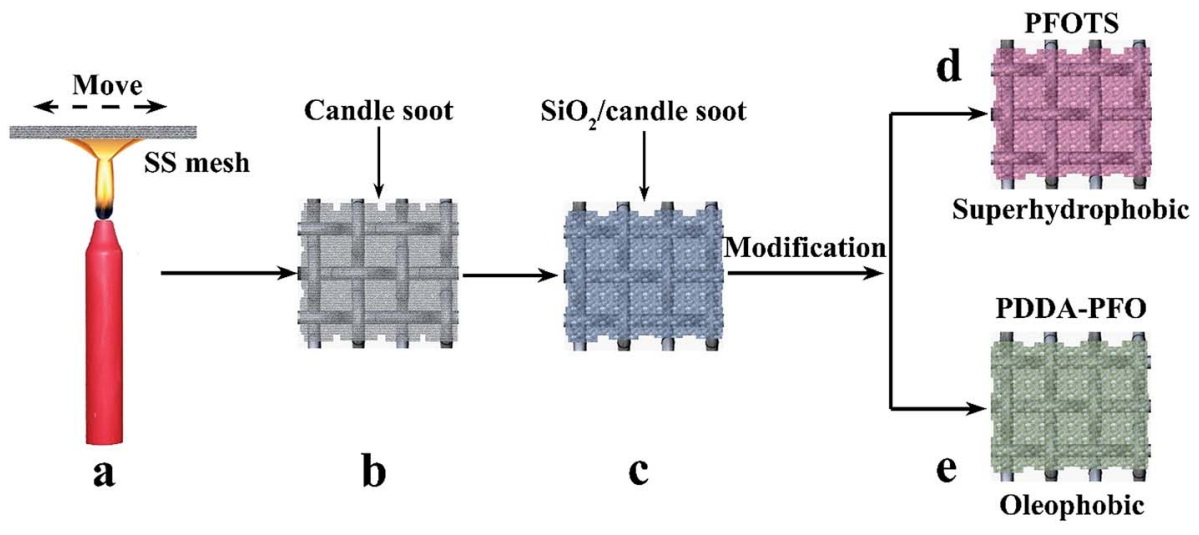

Fig. 1 Process of superhydrophobic and oleophobic mesh membranes preparation: (a) coating stainless steel mesh with carbon nanoparticle (candle soot), (b) carbon nanoparticle coated stainless steel mesh, (c) $\mathrm{SiO}_{2} /$ carbon stainless steel mesh, (d) $\mathrm{PFOTS} \mathrm{modified} \mathrm{SiO} /$ /carbon stainless steel mesh, (e) PDDA-PFO modified $\mathrm{SiO}_{2} /$ carbon stainless steel mesh. 
experiment was repeated 15 times. After each filtration, the oil or the water was collected for separation efficiency calculation.

The flux for separating oil or water was calculated with following equation:

$$
\text { Flux }=V / S t
$$

$V$ : the volume of oil or water filtrated per unit of time $\left(\mathrm{L} \mathrm{s}^{-1}\right) ; S$ : the projected area of the modified $\mathrm{SiO}_{2} /$ carbon stainless steel mesh membranes in contact with the oil/water mixture $\left(\mathrm{m}^{2}\right) ; t$ : filtration time (s).

The separation efficiency of oil or water was calculated with following equation:

$$
E=W_{\text {collected }} / W_{\text {original }}
$$

$E$ : separation efficiency; $W_{\text {collected }}$ the volume of collected oil or water after separation; $W_{\text {original }}$ : the volume of the original oil or water.

\subsection{Characterization}

The morphologies of the stainless steel mesh, carbon nanoparticle coated stainless steel mesh, $\mathrm{SiO}_{2} /$ carbon stainless steel mesh, both the PFOTS and PDDA-PFO modified $\mathrm{SiO}_{2} /$ carbon stainless steel meshes were examined by Nova 630 field emission scanning microscopy (FESEM). The chemical composition of the surface was analyzed by X-ray photoelectron spectroscopy (XPS, K-Alpha). High-resolution transmission electron microscopy (HRTEM) images were obtained on a Titan ST microscope (FEI Co.) operating at $300 \mathrm{kV}$. The surface wettability of the stainless steel mesh, carbon nanoparticle coated stainless steel mesh, $\mathrm{SiO}_{2} /$ carbon stainless steel mesh, PFOTS or PDDA-PFO modified $\mathrm{SiO}_{2} /$ carbon stainless steel meshes were characterized using an easy drop contact angle measurement instrument at ambient temperature in air.

\section{Results and discussion}

\subsection{Morphological evolution of the PFOTS or PDDA-PFO modified $\mathrm{SiO}_{2} /$ carbon stainless steel mesh membranes}

The surface morphologies of the stainless steel mesh, carbon nanoparticle coated stainless steel mesh, $\mathrm{SiO}_{2} /$ carbon stainless steel mesh, PFOTS or PDDA-PFO modified $\mathrm{SiO}_{2} /$ carbon stainless steel meshes were shown in Fig. 2a-e. Fig. 2a(I-III) present the typical morphology of the uncoated stainless steel mesh. The low magnified SEM image (Fig. 2a(I)) showed that the uncoated stainless steel mesh was knitted by stainless steel wires with an average pore size of about $45 \mu \mathrm{m}$. The surface of the stainless steel wires is relatively smooth except for some protrusions integrated on surface which maybe produced by abrasion (Fig. 2a(II) and (III)). During the candle soot-coating process, small carbon spherical particles with diameters of
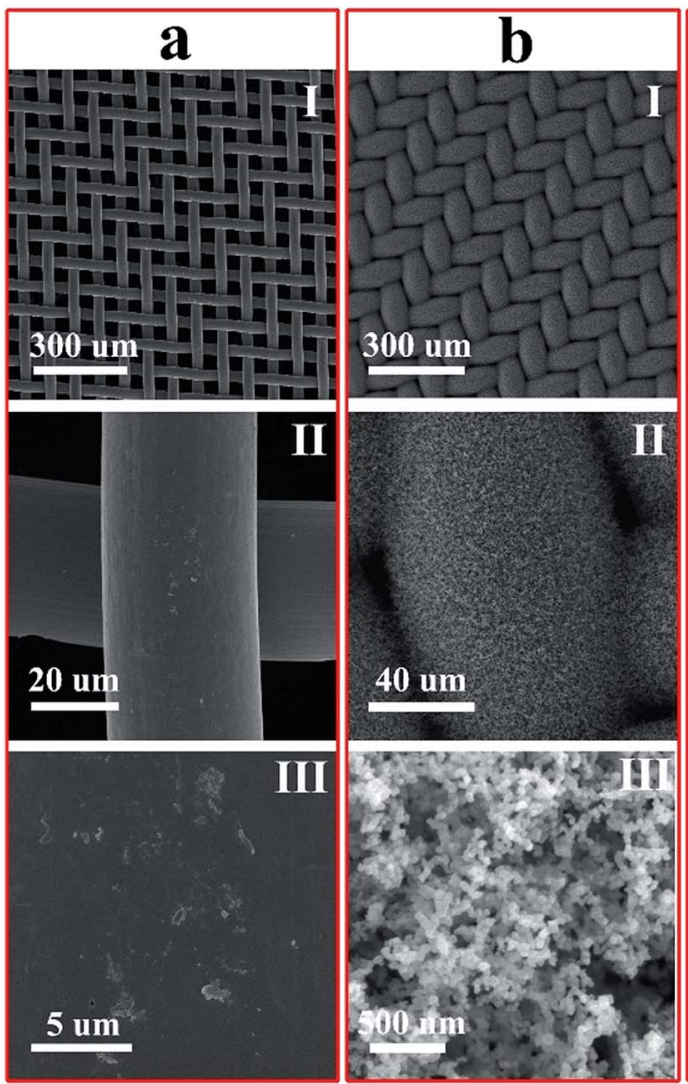
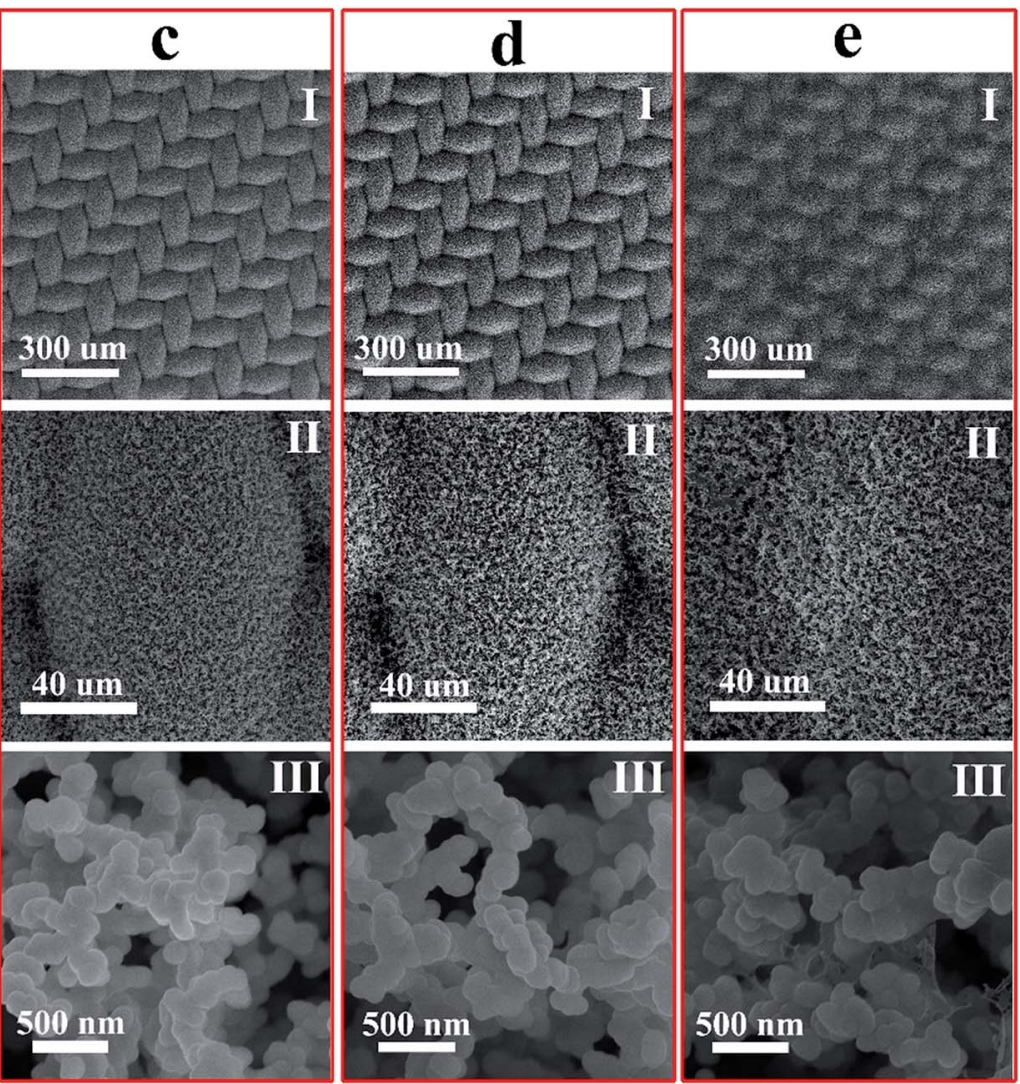

Fig. 2 SEM images of (a) blank stainless steel mesh, (b) carbon nanoparticle coated stainless steel mesh, (c) $\mathrm{SiO}_{2} / \mathrm{carbon}$ stainless steel mesh, (d) superhydrophobic $\mathrm{SiO}_{2}$ /carbon stainless steel mesh and (e) oleophobic $\mathrm{SiO}_{2}$ /carbon stainless steel mesh. 
tens of nanometers were generated because of the incomplete combustion of the candle soot (Fig. 2b(I) to (III)). The deposited carbon nanoparticles coagulated together to form an interconnected network on the stainless steel wire surface and the holes of stainless steel mesh were completely covered. Because this carbon nanoparticle layer is not stable and can easily be removed, ${ }^{41}$ a more stable and hydrophilic layer of $\mathrm{SiO}_{2}$ was further deposited on the surface of the as-obtained carbon nanoparticles. As shown in Fig. 2c(I) to (III), after being coated with $\mathrm{SiO}_{2}$, a hierarchical textured surface morphology with multiple scales of roughness and large numbers of protrusions was generated (Fig. 2c(III)). Comparing the size of the $\mathrm{SiO}_{2}$ (Fig. 2c(III)) formed on the surface of the carbon nanoparticles with that of original carbon nanoparticles (Fig. 2b(III)), the particle size increased approximately tenfold. Notably, the size and structure of these particles were very irregular, thus, increasing the total roughness of the surface. Moreover, the deposition of $\mathrm{SiO}_{2}$ also increased the hydrophilicity of the surface. As is well known, the solid surface wettability depends

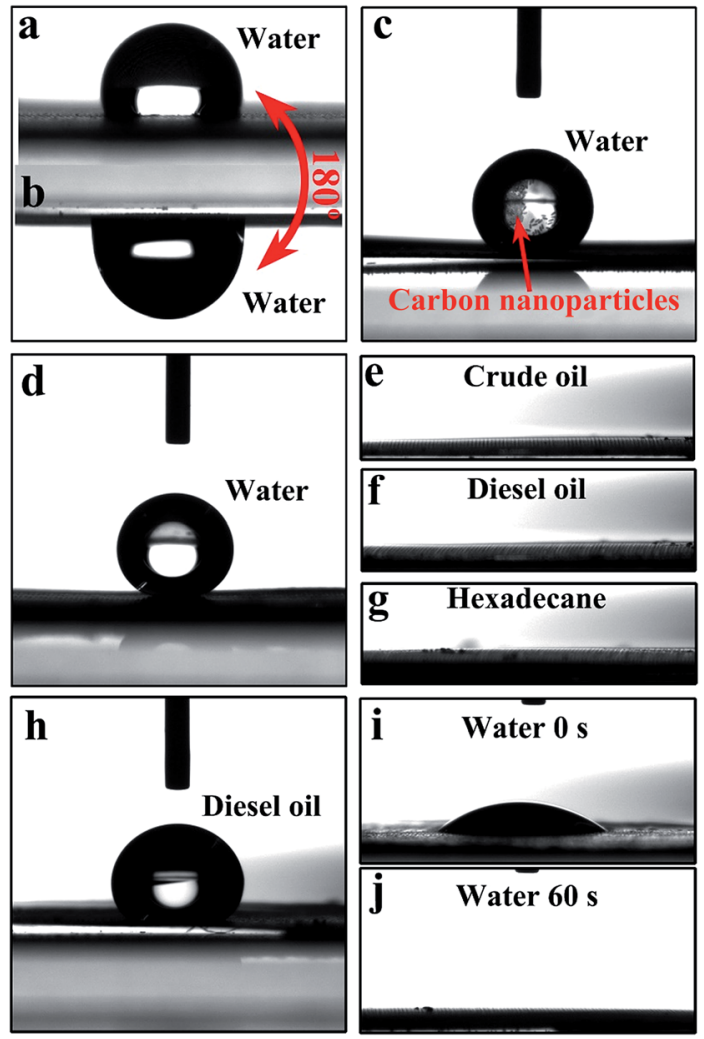

Fig. 3 Surface wettability of blank and modified stainless steel meshes: a water droplet $(8 \mu \mathrm{L})$ staying on the blank (a) and after tilting $180^{\circ}$ (b) stainless steel mesh, (c) a water droplet $(8 \mu \mathrm{L})$ staying on the carbon coated stainless steel mesh, $(\mathrm{d})$ a water droplet $(8 \mu \mathrm{L})$ staying on the PFOTS modified $\mathrm{SiO}_{2}$ /carbon stainless steel mesh, $(\mathrm{e}-\mathrm{g})$ droplets $(8 \mu \mathrm{L})$ of crude oil, diesel and hexadecane on the PFOTS modified $\mathrm{SiO}_{2} /$ carbon stainless steel meshes: oils will penetrate into the meshes as soon as they contact with the meshes, (h) a diesel oil droplet $(8 \mu \mathrm{L})$ staying on the PDDA-PFO modified $\mathrm{SiO}_{2} /$ carbon stainless steel mesh, (i) and (j) a water droplet contacting with PDDA-PFO modified $\mathrm{SiO}_{2} /$ carbon stainless steel mesh from 0 to $60 \mathrm{~s}$ : after $60 \mathrm{~s}$, water will penetrate into the oleophobic stainless steel mesh completely. on the roughness and chemical composition of the solid surface. $^{32}$ Therefore, the increase in surface roughness by creation a layer of non-uniform aggregates of $\mathrm{SiO}_{2} /$ carbon makes it possible to fabricate special superhydrophobic/ superoleophilic or oleophobic/superhydrophilic surfaces. To generate such special superhydrophobicity/superoleophilicity or oleophobicity/superhydrophilicity, the $\mathrm{SiO}_{2} /$ carbon stainless steel meshes were further modified with PFOTS and PDDA-PFO polymers, respectively. During the modification process, the hydrolyzed PFOTS or PDDA-PFO were attached onto the $\mathrm{SiO}_{2} /$ carbon surface. The XPS spectra were carried out to further provide the information that low-surface-energy PFOTS or PDDA-PFO was assembled onto $\mathrm{SiO}_{2}$ /carbon surface. In XPS spectra, the characteristic peak of fluorine (F) elements appearing at $688.7 \mathrm{eV}$ (both were showed in Fig. 2Sb and $\mathrm{c}^{\dagger}$ ) has confirmed that the original $\mathrm{SiO}_{2} /$ carbon can be successfully modified by PFOTS and PDDA-PFO. In addition, in Fig. $2 \mathrm{Sa}-\mathrm{c}, \dagger$ the presence of $\mathrm{Si} 2 \mathrm{~s}, \mathrm{Si} 2 \mathrm{p}$ peaks were all come from $\mathrm{SiO}_{2}$.

The combination of low energy fluorinated groups together with the alkyl groups of PFOTS silane resulted in a superhydrophobic/superoleophilic surface, while the fluorinated groups, together with the carboxyl and quaternary ammonium groups of PDDA-PFO resulted in an oleophobic/superhydrophilic surface..$^{15}$ More importantly, the modification does not change
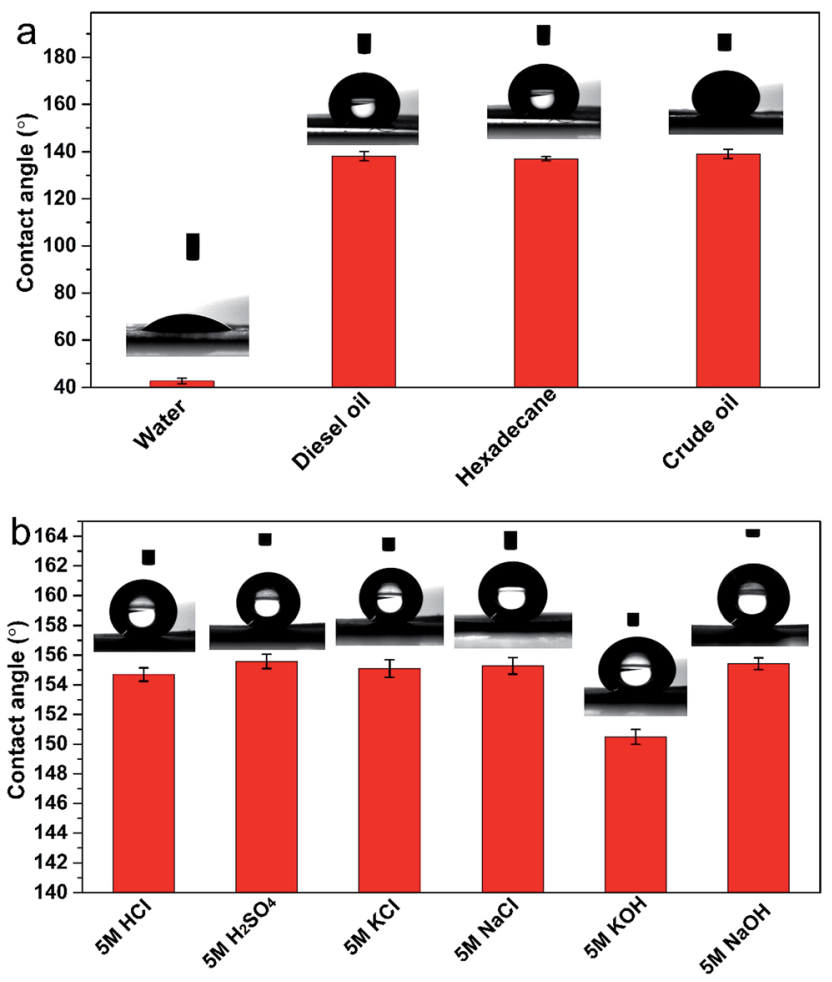

Fig. 4 (a) The contact angles of water and different oils on PDDA-PFO modified $\mathrm{SiO}_{2}$ /carbon stainless steel meshes. The inset shows the corresponding photos of water and oil droplets on the PDDA-PFO modified $\mathrm{SiO}_{2}$ /carbon stainless steel meshes, (b) the contact angles of different $5 \mathrm{M}$ acids, saturated salts and $5 \mathrm{M}$ bases on PFOTS modified $\mathrm{SiO}_{2} /$ carbon stainless steel meshes. The insets show the corresponding photos of different acids, salts and bases on PFOTS modified $\mathrm{SiO}_{2} /$ carbon stainless steel meshes, respectively. 
the surface morphology of the $\mathrm{SiO}_{2} /$ carbon stainless steel meshes. As shown in Fig. $2 \mathrm{~d}$ and e, the size and structure of the $\mathrm{SiO}_{2} /$ carbon nanoparticles are maintained after modification.

However there may be some variation in the final thicknesses of the coatings even though the candle soot and $\mathrm{SiO}_{2}$ coatings were made under the same conditions as it was created via a manual process. ${ }^{42}$

\subsection{The wettability of the superhydrophobic/} superoleophilic or oleophobic/superhydrophilic modified $\mathrm{SiO}_{2} /$ carbon stainless steel mesh membranes

The liquid wettability, including water and different oils, of the uncoated stainless steel mesh, carbon nanoparticle coated stainless steel mesh, $\mathrm{SiO}_{2} /$ carbon stainless steel mesh and PFOTS or PDDA-PFO modified $\mathrm{SiO}_{2}$ /carbon stainless steel meshes are shown in Fig. 3. Fig. 3a and b respectively present the shapes of a water droplet on an uncoated stainless steel mesh at a tilted angle of $0^{\circ}$ and $180^{\circ}$. Clearly, the uncoated stainless steel mesh shows hydrophobicity with a water CA of approximately $110^{\circ}$. However, the water droplet still maintains a semispherical shape without obvious distortion when the uncoated stainless steel mesh is gradually tilted to $180^{\circ}$, indicating that the uncoated mesh has a high CA hysteresis with respect to water droplets. Fig. 3c shows the photographs of a water droplet resting on the surface of carbon nanoparticles coated stainless steel mesh. The carbon nanoparticles coated stainless steel mesh also shows hydrophobicity with a water CA of about $138^{\circ}$. As we discussed above, carbon nanoparticles coated stainless steel mesh is unstable, and the carbon nanoparticles easily fall off. As shown in Fig. 3c, once the water droplet contacts the surface of the carbon nanoparticles coated stainless steel mesh, a large amount of carbon nanoparticles are absorbed by the water droplet (marked with a red arrow in Fig. 3c). Fig. 3d exhibits a water droplet resting on the PFOTS
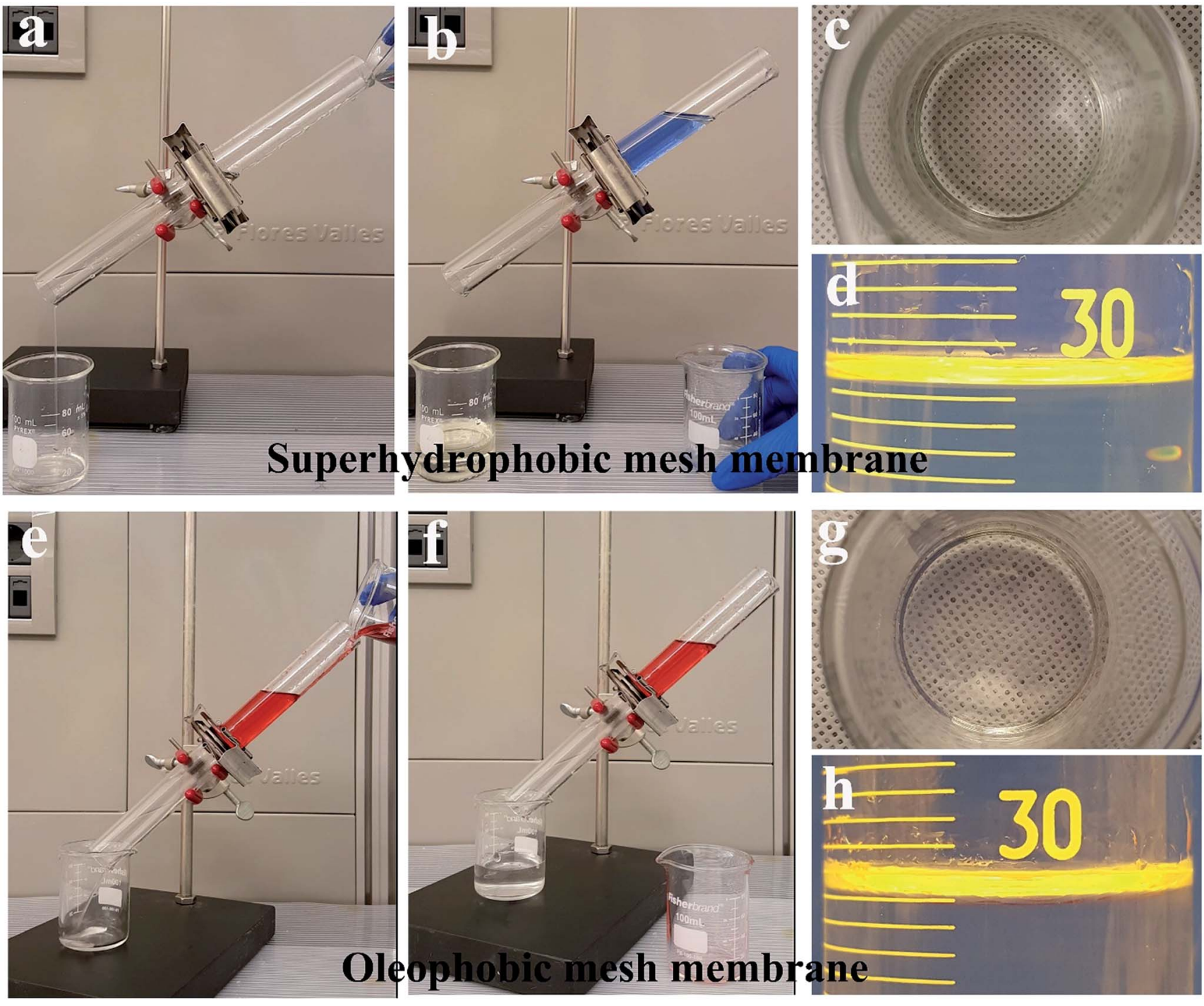

Fig. 5 The separation of oil/water with PFOTS modified ((a) and (b), water was dyed blue with methyl blue) and PDDA-PFO modified ((e) and (f), oil was dyed with Sudan III) $\mathrm{SiO}_{2}$ /carbon stainless steel meshes, (c) the top view of collected oil (no water was found), (d) the volume of collected oil, (g) the top view of collected water (no oil was found), and (h) the volume of collected water. 
modified $\mathrm{SiO}_{2} /$ carbon stainless steel mesh with very high CA (over $150^{\circ}$ ), while the oil droplets of crude oil (Fig. 3e), diesel oil (Fig. 3f) and hexadecane (Fig. 3g) rapidly spread and penetrate the PFOTS modified $\mathrm{SiO}_{2} /$ carbon stainless steel mesh as soon as they touch the upper surface of the coated mesh, clearly indicating the significant hydrophobicity and oleophilicity of the PFOTS modified $\mathrm{SiO}_{2} /$ carbon stainless steel mesh. The wettability of PDDA-PFO modified $\mathrm{SiO}_{2} /$ carbon stainless steel mesh is shown in Fig. $3 \mathrm{~h}-\mathrm{j}$. The PDDA-PFO modified $\mathrm{SiO}_{2} /$ carbon stainless steel mesh exhibits oleophobicity with a diesel oil CA of approximately $138^{\circ}$ (Fig. 3h). It is noteworthy that the water droplet also shows a certain CA of about $43^{\circ}$ as it was just placed onto the top of the PDDA-PFO modified $\mathrm{SiO}_{2} /$ carbon stainless steel mesh (Fig. 3i). The CA of the water gradually decreased with time, and after approximately $60 \mathrm{~s}$, the water droplet spread over the PDDA-PFO modified $\mathrm{SiO}_{2} /$ carbon stainless steel mesh (Fig. 3j). It proved that the surface of PDDA-PFO modified $\mathrm{SiO}_{2} /$ carbon stainless steel mesh becomes superhydrophilic. This is a result of water-induced surface molecular rearrangement and the three dimensional capillary effect: water completely fills all of the surface asperities to form a fully wetted interface. ${ }^{15}$ The wettability of crude oil and hexadecane were also examined as shown in Fig. 4a. As expected, for crude oil and hexadecane PDDA-PFO modified $\mathrm{SiO}_{2} /$ carbon stainless steel mesh also exhibited oleophobicity with a CA greater than $140^{\circ}$.

Additionally, the stability of the PFOTS modified $\mathrm{SiO}_{2} /$ carbon stainless steel mesh was evaluated under a series of harsh environment including different acidic, basic and high salt conditions. The wetting behaviors of some common strong acids ( $5 \mathrm{M}$ of $\mathrm{HCl}$ and $\mathrm{H}_{2} \mathrm{SO}_{4}$ ), strong bases $(5 \mathrm{M}$ of $\mathrm{KOH}$ and $\mathrm{NaOH}$ ) as well as saturated salts (5 $\mathrm{M}$ of $\mathrm{NaCl}$ and $\mathrm{KCl}$ ) were also examined as can be seen in Fig. 4b. Interestingly, the PFOTS modified $\mathrm{SiO}_{2} /$ carbon stainless steel mesh still exhibits excellent repellency for all the tested strong acids, strong bases and saturated salts, indicating a good stability of PFOTS modified $\mathrm{SiO}_{2} /$ carbon nanoparticle coated stainless steel mesh under a series of harsh environment.

\subsection{Separation of oil/water mixture with superhydrophobic/ superoleophilic or oleophobic/superhydrophilic modified $\mathrm{SiO}_{2} /$ carbon stainless steel mesh membranes}

With different surface wettability membranes, different oil/water mixtures can be selectively separated. The superhydrophobic/ superoleophilic $\mathrm{SiO}_{2} /$ carbon stainless steel meshes can be used to separate a small amount of water from a large amount of oil to increase the separation efficiency. The superhydrophobic/ superoleophilic $\mathrm{SiO}_{2} /$ carbon stainless steel mesh acting as the separation membrane was mounted between two glass tubes. The diesel/water mixture (water was dyed blue with methyl blue for the purpose of enhancing the visual effect) was poured into the upper glass tube for the separation process. As shown in Fig. $5 \mathrm{a}$ and $\mathrm{b}$, the diesel oil immediately permeated through the superhydrophobic/superoleophilic $\mathrm{SiO}_{2} /$ carbon stainless steel mesh and flowed down under the influence of gravity due to its oleophilicity. Meanwhile, water was still retained above the membrane due to its superhydrophobicity. After separation, no water was observed in the collected oil (Fig. 5c) and almost $30 \mathrm{~mL}$ of oil can be collected (Fig. 5d). The flux of the oil that permeated through the superhydrophobic/superoleophilic $\mathrm{SiO}_{2} /$ carbon stainless steel mesh membrane was measured by calculating the flow volume in the unit time from the valid area (eqn (1)). As shown in Fig. 6a, the superhydrophobic/superoleophilic $\mathrm{SiO}_{2} /$ carbon stainless steel mesh membrane exhibits high water flux over $934 \mathrm{~L} \mathrm{~m}^{-2} \mathrm{~h}^{-1}$ in all measured time.

In contrast, the oleophobic/superhydrophilic $\mathrm{SiO}_{2} /$ carbon stainless steel meshes can be used to separate a small amount of oil from a large amount of water to increase the separation efficiency. The oleophobic/superhydrophilic $\mathrm{SiO}_{2} /$ carbon stainless steel mesh, acting as the separation membrane, was mounted between two glass tubes. The as-prepared diesel/ water mixture (diesel oil was dyed red with Sudan III for the purpose of enhancing the visual effect) was poured into the upper glass tube for separation process. As shown in Fig. 5e and $f$, the water immediately permeated through the oleophobic/superhydrophilic $\mathrm{SiO}_{2} /$ carbon stainless steel mesh and flowed down only under the influence of gravity due to its superhydrophilicity. Meanwhile, diesel oil was still retained above the membrane due to its oleophobicity. After separation, no diesel oil was observed in the separated water (Fig. 5g), and we can collect almost $30 \mathrm{~mL}$ of water (Fig. 5h). The flux of the water that permeated through the oleophobic/
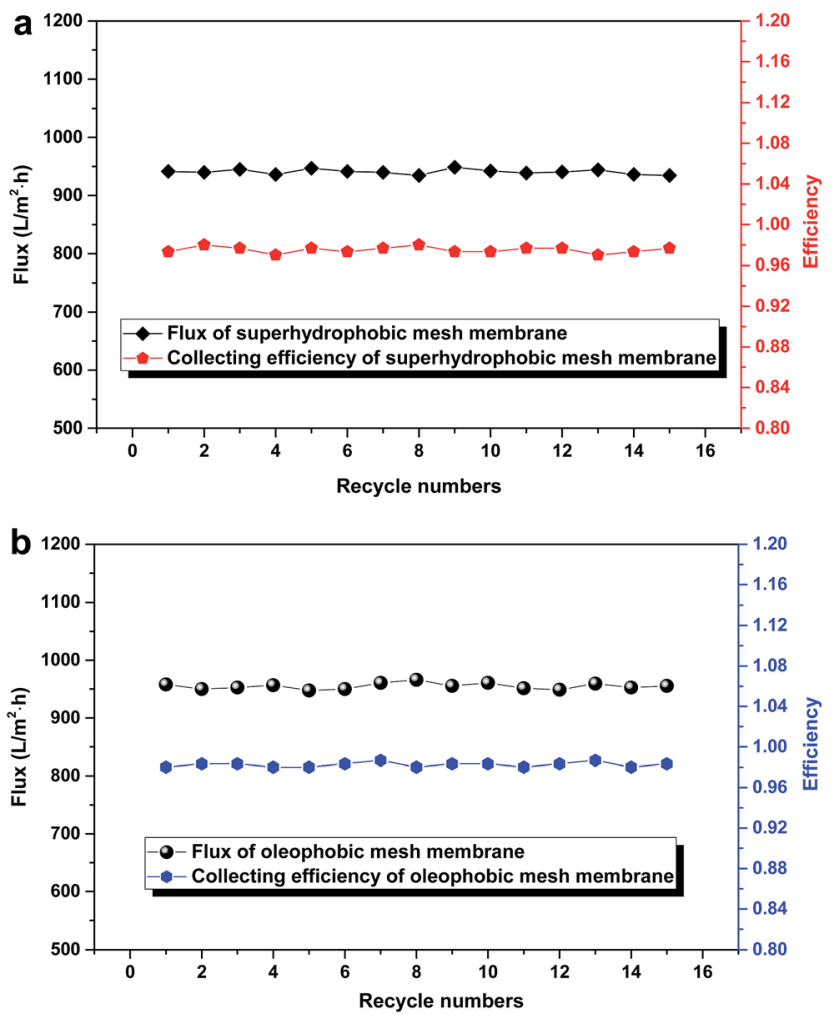

Fig. 6 Study of the stability of the as-fabricated superhydrophobic PFOTS and oleophobic PDDA-PFO modified $\mathrm{SiO}_{2} /$ carbon stainless steel meshes, the change of the flux and rate of collected oil or water to the original oil or water with increasing cycle number, respectively. 
superhydrophilic $\mathrm{SiO}_{2} /$ carbon stainless steel mesh membrane was measured by calculating the flow volume in the unit time from the valid area (eqn (1)). As shown in Fig. 6b, the oleophobic/superhydrophilic $\mathrm{SiO}_{2} /$ carbon stainless steel mesh membrane exhibits high water flux over $947 \mathrm{~L} \mathrm{~m}^{-2} \mathrm{~h}^{-1}$ in all measured time.

The separation efficiency for both separation processes was calculated according to eqn (2). After each separation, the oil or water was collected in the containers and measured. ${ }^{\mathbf{2 4 , 2 8 , 4 3}}$ The separation efficiencies obtained were repeatedly $97 \%$ for all of the repeated experiments, indicating high separation efficiency. Most importantly, the flux and efficiency for all of the repeated oil/water separations remained the same after 15 cycles without any noticeable deterioration. This suggests that both the superhydrophobic/superoleophilic and oleophobic/superhydrophilic $\mathrm{SiO}_{2}$ /carbon stainless steel mesh membranes possess the virtue of stability, durability and reusability.

\section{Conclusions}

In conclusion, superhydrophobic/superoleophilic or oleophobic/ superhydrophilic stainless steel mesh membranes were fabricated via a simple candle soot coating, the template of carbon nanoparticles with diameters of approximately ten nanometers was first collected on the surface of steel wires based on a candle soot coating process and then a more stable layer of $\mathrm{SiO}_{2}$ was deposited onto the template via chemical vapor deposition. After being modified with special wettability polymers of PFOTS or PDDA-PFO, the expected superhydrophilic/superoleophilic or oleophobic/superhydrophilic mesh membranes were produced. Such membranes can be selected to remove oils or water for different purposes to increase the separation efficiency. The membranes exhibit a high separation flux of more than $930 \mathrm{~L}$ $\mathrm{m}^{-2} \mathrm{~h}^{-1}$ and a collecting efficiency over $97 \%$. Most decisively, the repetitions of separation indicate that these superhydrophobic/ superoleophilic or oleophobic/superhydrophilic mesh membranes are durable, stable and reusable, making them good candidates for treating real oil-polluted water in different practical applications as well as in oil spill cleanups.

\section{Acknowledgements}

This work was supported by science and Technology Planning project of Guangdong (No. 2014A010105041, 2013B021700001), China. We also acknowledge Casey Wetzel from U. M. and JeanPierre de Levay from U. W. for comments that greatly improved the manuscript.

\section{References}

1 L. Gossen and L. Velichkina, Pet. Chem., 2006, 46, 67-72.

2 M. Schrope, Nature, 2011, 472, 152-154.

3 M. Cheryan and N. Rajagopalan, J. Membr. Sci., 1998, 151, 13-28.

4 M. A. Shannon, P. W. Bohn, M. Elimelech, J. G. Georgiadis,

B. J. Mariñas and A. M. Mayes, Nature, 2008, 452, 301-310.

5 S. Elmaleh and N. Ghaffor, J. Membr. Sci., 1996, 118, 111-120.
6 R. A. Kerr, Science, 2010, 329, 735-736.

7 H. W. Liang, Q. F. Guan, L. F. Chen, Z. Zhu, W. J. Zhang and S. H. Yu, Angew. Chem., Int. Ed., 2012, 51, 5101-5105.

8 C. Teng, X. Lu, G. Ren, Y. Zhu, M. Wan and L. Jiang, Adv. Mater. Interfaces, 2014, 1, 1400099.

9 Z. Y. Wu, C. Li, H. W. Liang, J. F. Chen and S. H. Yu, Angew. Chem., Int. Ed., 2013, 52, 2925-2929.

10 Z.-Y. Wu, C. Li, H.-W. Liang, Y.-N. Zhang, X. Wang, J.-F. Chen and S.-H. Yu, Sci. Rep., 2014, 4, 4097.

11 J. Li, L. Yan, X. Tang, H. Feng, D. Hu and F. Zha, Adv. Mater. Interfaces, 2016, 3, 1500770.

12 C. Ruan, K. Ai, X. Li and L. Lu, Angew. Chem., Int. Ed., 2014, 53, 5556-5560.

13 Z. Xu, K. Miyazaki and T. Hori, Adv. Mater. Interfaces, 2015, 2, 1500255.

14 A. K. Kota, G. Kwon, W. Choi, J. M. Mabry and A. Tuteja, Nat. Commun., 2012, 3, 1025.

15 J. Yang, Z. Zhang, X. Xu, X. Zhu, X. Men and X. Zhou, J. Mater. Chem., 2012, 22, 2834-2837.

16 Y. Yu, H. Chen, Y. Liu, V. S. Craig, L. H. Li, Y. Chen and A. Tricoli, Polymer, 2014, 55, 5616-5622.

17 W. Zhang, Z. Shi, F. Zhang, X. Liu, J. Jin and L. Jiang, Adv. Mater., 2013, 25, 2071-2076.

18 F. Wang, S. Lei, Y. Xu and J. Ou, ChemPhysChem, 2015, 16, 2237-2243.

19 S. Wang, Y. Song and L. Jiang, Nanotechnology, 2006, 18, 015103.

20 C. Gao, Z. Sun, K. Li, Y. Chen, Y. Cao, S. Zhang and L. Feng, Energy Environ. Sci., 2013, 6, 1147-1151.

21 S. Li, J. Huang, M. Ge, C. Cao, S. Deng, S. Zhang, G. Chen, K. Zhang, S. S. Al-Deyab and Y. Lai, Adv. Mater. Interfaces, 2015, 2, 1500220.

22 B. Wang, Y. Liu, Y. Zhang, Z. Guo, H. Zhang, J. H. Xin and L. Zhang, Adv. Mater. Interfaces, 2015, 2, 1500234.

23 F. Zhang, W. B. Zhang, Z. Shi, D. Wang, J. Jin and L. Jiang, Adv. Mater., 2013, 25, 4192-4198.

24 Y. Yu, H. Chen, Y. Liu, V. Craig, L. H. Li and Y. Chen, Adv. Mater. Interfaces, 2014, 1, 1300002.

25 Y. Yu, H. Chen, Y. Liu, V. S. Craig, C. Wang, L. H. Li and Y. Chen, Adv. Mater. Interfaces, 2015, 2, 1400267.

26 Q. Wen, J. Di, L. Jiang, J. Yu and R. Xu, Chem. Sci., 2013, 4, 591-595.

27 R. Yang, P. Moni and K. K. Gleason, Adv. Mater. Interfaces, 2015, 2, 1400489.

28 J. Zeng and Z. Guo, Colloids Surf., A, 2014, 444, 283-288.

29 Y. Dong, J. Li, L. Shi, X. Wang, Z. Guo and W. Liu, Chem. Commun., 2014, 50, 5586-5589.

30 Y. Huang, H. Li, L. Wang, Y. Qiao, C. Tang, C. Jung, Y. Yoon, S. Li and M. Yu, Adv. Mater. Interfaces, 2015, 2, 1400433.

31 B. Cortese, D. Caschera, F. Federici, G. M. Ingo and G. Gigli, J. Mater. Chem. A, 2014, 2, 6781-6789.

32 J. Li, D. Li, Y. Yang, J. Li, F. Zha and Z. Lei, Green Chem., 2016, 18, 541-549.

33 L. Feng, S. Li, Y. Li, H. Li, L. Zhang, J. Zhai, Y. Song, B. Liu, L. Jiang and D. Zhu, Adv. Mater., 2002, 14, 1857-1860.

34 J. Li, R. Kang, X. Tang, H. She, Y. Yang and F. Zha, Nanoscale, 2016, 8, 7638-7645. 
35 J. Li, L. Yan, H. Li, W. Li, F. Zha and Z. Lei, J. Mater. Chem. A, 2015, 3, 14696-14702.

36 Y. Liu, Y. Zhang, X. Fu and H. Sun, ACS Appl. Mater. Interfaces, 2015, 7, 20930-20936.

37 T. Huang, L. Zhang, H. Chen and C. Gao, J. Mater. Chem. A, 2015, 3, 19517-19524.

38 S. Ramos, J. Dias and B. Canut, J. Colloid Interface Sci., 2015, 440, 133-139.

39 C. Crick, J. Gibbins and L. Parkin, J. Mater. Chem. A, 2013, 1, 5943-5948.
40 L. Zhi Chen, R. Wang, C. Guo, P. Zhang and S. Pang, Appl. Surf. Sci., 2012, 258, 8970-8973.

41 K. Seo and M. Kim, Carbon, 2014, 68, 583-596.

42 M. Paven, P. Papadopoulos, S. Schöttler, X. Deng, V. Mailänder, D. Vollmer and H.-J. Butt, Nat. Commun., 2013, 4, 2512.

43 Q. Pan, M. Wang and H. Wang, Appl. Surf. Sci., 2008, 254, 6002-6006. 